\title{
Resultados académicos y desarrollo cognitivo en un programa de inmersión dirigido a escolares de nivel socio-cultural bajo
}

\author{
JOSEP MARÍA SERRA \\ Universidad de Barcelona

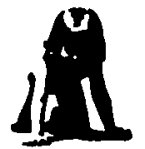 \\ Resumen
}

Este artículo bace un repaso de las principales caracteristicas de los programas de inmersión asi como de las bases psicopedagógicas en las que se fundamentan, encontrándose argumentos a favor de la aplicación de este tipo de programas a escolares con bajo nivel socio-cultural. Esta investigación ba llevado a cabo la evaluacion y seguimiento al final del ciclo inicial de una muestra de alumnos que empezaron a los 4 años un programa de inmersión realizado en una escuela situada en el barrio del Carmel de Barcelona y ba comparado los resultados obtenidos con los de otra escuela, situada en el mismo barrio, que realiza la enseñanza en la lengua familiar de los alumnos (castellano).

Palabras clave: Bilingüismo, Inmersión, Adquisición de una segunda lengua, Interacción, Desarrollo cognitivo, Competencia lingüística.

\section{Academic results and cognitive development in an immersion programme for school children of a low sociocultural status}

\begin{abstract}
This paper reviews the main characteristics of inmersion programmes and the psychoeducational basis on which they are founded, finding arguments in favour of the application of these type of programmes to schoolchildren with a low sociocultural status. In this study, a sample of students, who bad begun an inmersion programme at the age of four, were assessed and followed up to the time when they reached second grade. The inmersion program. me was carried out in a school located in the Carmel district of Barcelona. The results were compared with those obtained at another school situated in the same area where instruction is ca. rried out in the home language of the students (Spanish).
\end{abstract}

Key words: Bilingualism, Inmersion, Adquisition of a second language, Cognitive development, Linguistic competence.

Dirección del autor: ICE de la Universidad de Barcelona. Plaza de la Universidad 1, 08007 Barcelona.

Original recibido: Marzo 1989. Revisión recibida: Abril 1989. Aceptado: Mayo 1989. 


\section{INTRODUCCION}

En los últimos diez años ha habido en Cataluña un incremento notable de los programas de cambio de lengua hogar-escuela, normalmente conocidos como programas de inmersión. Estos se corresponden con los programas de bilingüismo total y se han mostrado hasta ahora como los más potentes para animar la competencia lingüística en la segunda lengua. Su característica más importante reside en que son programas que van dirigidos al conjunto de la comunidad y no solamente a las minorías, y su objetivo consiste en conseguir que todos los miembros de una determinada comunidad dominen las lenguas que están presentes en el medio. En consecuencia, este tipo de programas incide de forma preferencial al inicio de la escolaridad en la lengua minoritaria o L2 lo cual implica, para muchos escolares, un cambio de lengua del hogar a la escuela.

Las bases de eŝte tipo de programas son las siguientes. En primer lugar, la voluntariedad en la asistencia. Esto es, los programas de inmersión escolarizan a alumnos cuyos padres desean que sus hijos aprendan una segunda lengua y reconocen que éste es el mejor sistema. En segundo lugar, estos programas cuentan con un profesorado bilingiue, conocedor de la lengua de los alumnos, de manera que se garantiza desde el primer momento que la comunicación en el aula no se interrumpa. Por otra parte, este planteamiento supone un enorme respeto por la lengua del alumno ya que nunca se le fuerza a que produzca en la segunda lengua. Por el contrario, se parte de la premisa de que la producción emerge tras la comprensión. La tercera característica es que todos los alumnos parten de un mismo punto en relación a la lengua de la escuela: la desconocen. Es decir, en los programas de inmersión, en la medida que el maestro/a presupone que el alumno no conoce la lengua que posteriormente será vehicular de contenidos centra su tarea en enseñarla. Esto sólo es posible desde un planteamiento pedagógico en el que la enseñanza de la segunda lengua se asemeja, por lo menos en sus actividades, al proceso de adquisición de su lengua familiar. De esta manera, el profesorado tiene que recurrir a las situaciones comunicativas en las que el escolar recibe un importante soporte contextual para negociar y compartir los significados necesarios para regular y controlar las tareas interactivas que se le proponen (Vila, 1985).

Por otra parte, uno de los objetivos de la escuela es transmitir los conocimientos que, generación tras generación, la especie humana ha ido adquiriendo a través de sus relaciones para dominar y transformar la naturaleza. Este objetivo se consigue empleando un conjunto de instrumentos compartidos por enseñantes y escolares, de los cuales el lenguaje es uno de los más importantes. Pero el instrumento lingüístico no existe de forma única sino que, contrariamente, se nos puede presentar de diferentes formas (código oral, escrito, etc.), requiriéndose habilidades diferentes, aunque no excluyentes, para dominar cada una de éstas. Así, la escuela emplea fundamentalmente la lectura y la escritura, cuyo dominio se convierte para el escolar en una parte muy importante de su éxito o fracaso escolar. Si estamos de acuerdo en que, 
como han demostrado numerosos trabajos sobre lecto-escritura, el desarrollo de las habilidades necesarias para encarar la lectura y la escritura reside en gran medida en las habilidades desarrolladas en relación al código oral, es obvia la enorme importancia que tiene el trabajo que se desarrolla en las aulas de parvulario y, más aún, en aquellas aulas donde la lengua de la escuela, la lengua que posteriormente será vehicular de contenidos, es para la mayoría de los alumnos desconocida y, además, el único lugar donde podrán aprenderla.

En la actualidad, hay en Cataluña cerca de 45.000 alumnos escolarizados en este tipo de programas y la mayoría de las escuelas a las que asisten están incluidas en zonas donde se llevan a cabo Planes Intensivos de Normalización. Esta consideración es importante, ya que buena parte de estos niños y niñas viene de ámbitos bastante deprivados socioeconómicamente y arrastran en muchas ocasiones un abanico considerable de problemas de índole social. Muchos de ellos llegan a la escuela con una baja competencia en su lengua materna y con un lenguaje muy desestructurado. De esta manera, los factores socioculturales se entrecruzan con los factores lingüísticos y pedagógicos. En estas condiciones, un programa de cambio de lengua hogar-escuela pudiera parecer contraproducente en la medida en que impediría el desarrollo de las habilidades ingüísticas necesarias para afrontar con éxito las tareas académicas. Sin embargo, de acuerdo con la hipótesis de Interdependencia Lingüística (Cummins, 1979; Vila, 1983), en la medida en que estos programas desarrollarían una competencia lingüística en la L2 no sólo no impedirían el desarrollo de las habilidades generales, sino que ayudarían a estructurar la lengua familiar de estos alumnos. Justamente, en este trabajo pretendemos evaluar esta afirmación tras el estudio de un programa de inmersión dirigido a niños y niñas de nivel sociocultural bajo.

\section{METODO}

\section{Muestra}

En este estudio hemos empleado una muestra de 80 alumnos de segundo de EGB repartidos en 4 grupos. Los dos primeros, grupo A y grupo $B$, con 16 y 17 sujetos respectivamente, seguían un programa de inmersión en catalán en una escuela y, los otros dos, grupo $C$ y grupo $\mathrm{D}$, con 24 y 23 sujetos, realizaban la enseñanza en su lengua materna (castellano) en la otra escuela. De la muestra original se eliminaron 5 sujetos (1 del grupo B, 2 del grupo $C$ y 1 del grupo D) que por diversos motivos no pudieron completar todas las pruebas, con lo cual el número de sujetos por grupo quedó de la siguiente manera: grupo A, 16; grupo B, 16; grupo C, 21 y grupo D, 22. Las dos escuelas están situadas en el barrio del Carmel, una de las zonas más deprivadas social y económicamente, de Barcelona. Los alumnos que asisten a éstas provienen de familias de nivel sociocultural bajo y muy bajo, nacidos en Barcelona, la mayoría de los cuales tienen el castellano como lengua materna. 


\section{Procedimiento}

Para evaluar los resultados, se ha seleccionado una batería de pruebas elaboradas por el Departament de Pedagogia Experimental de la Facultad de Filosofía i Ciències de l'Educació de la Universitat de Barcelona. Estas pruebas (vocabulario, lectura silenciosa, velocidad lectora, errores en la lectura, expresión escrita y comprensión oral) fueron complementadas por un test de inteligencia (Raven), una prueba de matemáticas y otra de experiencias elaboradas conjuntamente por las maestras de ambas escuelas en función de la programación de cada escuela y de los mínimos establecidos por el Departament d'Ensenyament de la Generalitat de Cataluña. Todas las pruebas se pasaron durante el mes de junio del curso escolar 1986-1987. La prueba de experiencias fue rehusada por mostrarse irrelevante para los objetivos de nuestro estudio. Igualmente, las pruebas de vocabulario, lectura silenciosa y comprensión oral se redujeron a la mitad después de haber efectuado un análisis de dificultad de los ítems y una división del test en dos mitades a partir de los datos resultantes de la administración de las mismas pruebas en una investigación realizada por Arnau (1985).

Para la evaluación y posterior análisis de los datos hemos utilizado el paquete de programas de análisis estadísticos MICROSTA. Se obtuvieron los parámetros descriptivos y se realizaron anovas de tipo factorial para datos independientes. El número de variables analizadas ha sido 8 (LS: Lectura Silenciosa; CO: Comprensión Oral; V: Vocabulario; VLEC: Velocidad Lectora; ELEC: Errores en la Lectura; EX: Expresión Escrita; MAT: Matemáticas y CI: Cociente Intelectual) y el número de grupos 4, siendo los grupos A y B los experimentales y los grupos $\mathrm{C}$ y $\mathrm{D}$ los grupos control.

\section{RESULTADOS Y DISCUSION}

Exponemos a continuación los resultados obtenidos. En primer lugar comentaremos los resultados del análisis descriptivo de cada grupo respecto a las 8 variables del estudio y, en el segundo, analizaremos los datos comparativos entre los grupos.

\section{Descriptivos}

Una primera observación de los datos (ver Tabla 1) nos muestra que el grupo B de inmersión obtiene unas medias superiores a los otros grupos en todas las pruebas, exceptuando la de velocidad lectora (inferiores a los grupos $C$ y $D$ que realizan la enseñanza en castellano) y la de errores en la lectura (superior a los grupos $\mathrm{C}$ y D). La segunda observación hace referencia al hecho de que el grupo $\mathrm{A}$ obtiene unas medias ligeramente superiores en las pruebas de comprensión oral, lectura silenciosa y matemáticas respecto a los grupos $C$ y $D$. En las pruebas de vocabulario y de velocidad lectora las medias son inferiores respecto de los mismos grupos. La tercera observación se deriva del análisis de 
TABla I

Descriptivos de las variables estudiadas en cada uno de los grupos

Grupo A

\begin{tabular}{lcrrrc}
\hline Variable & N & Media & $\begin{array}{c}\text { Desviación } \\
\text { típica }\end{array}$ & Minimum & Maximum \\
\hline LS & 16 & 7.5625 & 4.3433 & 0.0000 & 14.0000 \\
CO & 16 & 9.5000 & 2.7809 & 4.0000 & 14.0000 \\
V & 16 & 13.3750 & 4.9649 & 4.0000 & 21.0000 \\
MAT & 16 & 16.0000 & 8.5323 & 1.0000 & 27.0000 \\
VLEC & 16 & 34.7500 & 21.4025 & 0.0000 & 78.0000 \\
ELEC & 16 & 16.9375 & 12.9535 & 1.0000 & 39.0000 \\
EX & 16 & 27.0000 & 15.0022 & 10.0000 & 66.0000 \\
CI & 16 & 57.0625 & 9.6019 & 46.0000 & 81.0000 \\
\hline
\end{tabular}

Grupo B

\begin{tabular}{lllrrr}
\hline Nombre & N & Media & $\begin{array}{c}\text { Desviación } \\
\text { típica }\end{array}$ & Minimum & Maximum \\
\hline LS & 16 & 10.1250 & 3.4424 & 3.0000 & 14.0000 \\
CO & 16 & 11.3125 & 1.8518 & 7.0000 & 13.0000 \\
V & 16 & 20.0625 & 3.7854 & 14.0000 & 27.0000 \\
MAT & 16 & 22.7500 & 4.0579 & 16.0000 & 30.0000 \\
VLEC & 16 & 43.1875 & 15.7997 & 19.0000 & 78.0000 \\
ELEC & 16 & 10.2500 & 9.4340 & 0.0000 & 38.0000 \\
EX & 16 & 21.9375 & 11.6010 & 0.0000 & 43.0000 \\
CI & 16 & 62.8750 & 16.1240 & 44.0000 & 95.0000 \\
\hline
\end{tabular}

Grupo C

\begin{tabular}{lcrrrr}
\hline Variable & N & \multicolumn{1}{c}{ Media } & $\begin{array}{c}\text { Desviación } \\
\text { típica }\end{array}$ & Minimum & Maximum \\
\hline LS & 21 & 7.0476 & 2.9236 & 2.0000 & 12.0000 \\
CO & 21 & 9.6667 & 2.2435 & 6.0000 & 14.0000 \\
V & 21 & 18.6667 & 4.1150 & 10.0000 & 26.0000 \\
MAT & 21 & 15.4762 & 6.6513 & 3.0000 & 28.0000 \\
VLEC & 21 & 46.9524 & 15.3019 & 0.0000 & 71.0000 \\
ELEC & 21 & 9.6667 & 6.6056 & 0.0000 & 25.0000 \\
EX & 21 & 27.2957 & 13.1801 & 3.0000 & 52.0000 \\
CI & 21 & 64.3810 & 12.2208 & 44.0000 & 92.0000 \\
\hline
\end{tabular}

Grupo D

\begin{tabular}{lcrrrr}
\hline Variable & N & \multicolumn{1}{c}{ Media } & $\begin{array}{c}\text { Desviación } \\
\text { típica }\end{array}$ & Minimum & Maximum \\
\hline LS & 22 & 7.0000 & 3.4778 & 0.0000 & 13.0000 \\
CO & 22 & 8.5455 & 3.0194 & 2.0000 & 12.0000 \\
V & 22 & 17.9545 & 5.6356 & 0.0000 & 25.0000 \\
MAT & 22 & 15.8636 & 8.2652 & 0.0000 & 29.0000 \\
VLEC & 22 & 44.0455 & 15.3969 & 18.0000 & 78.0000 \\
ELEC & 22 & 9.5909 & 11.6319 & 0.0000 & 46.0000 \\
EX & 22 & 20.1364 & 10.9731 & 4.0000 & 41.0000 \\
CI & 22 & 61.0455 & 12.2065 & 41.0000 & 95.0000 \\
\hline
\end{tabular}


las desviaciones estándar. Así, por una parte, se observa que el grupo $\mathrm{C}$ se revela como el grupo más homogéneo de todos al obtener las desviaciones más bajas, exceptuando las pruebas de vocabulario y expresión escrita. Por la otra, en las pruebas de velocidad lectora y expresión escrita es donde se observan las desviaciones más altas en los 4 grupos. Este hecho matiza la valoración en términos absolutos de estos datos. Es decir, a pesar de que nuestros niños estén al final del ciclo inicial y ya lleven varios años introducidos en el aprendizaje de la lectura y la escritura, aún están en las primeras etapas de dicha adquisición, apareciendo, por tanto, importantes diferencias individuales. Por eso, no es extraño que en las pruebas de velocidad lectora y de expresión escrita, aquellas que reflejan mejor dichas diferencias, aparezca una desviación más amplia. La normalidad de este fenómeno es, justamente, su aparición en los cuatro grupos.

\section{Comparación entre grupos}

En cuanto al segundo bloque de resultados, derivados de los análisis de varianza entre los grupos, tenemos que destacar tres datos significativos (ver Tablas 2 a 6 ):

a) No existe diferencia significativa entre los grupos respecto al cociente intelectual (CI).

b) El grupo B de inmersión obtiene unos resultados significativamente superiores respecto a los grupos $\mathrm{A}, \mathrm{C}$ y $\mathrm{D}$ en las pruebas de comprensión oral (CD), lectura silenciosa (LS) y matemáticas (MAT).

c) El grupo A de inmersión obtiene unos resultados significativamente inferiores respecto a los grupos $B, C$ y $D$ en la prueba de vocabulario (V).

Estos resultados tienen, a nuestro entender, una enorme importancia en nuestro estudio. Pero, antes de interpretarlos, creemos necesario añadir dos puntos más procedentes del análisis descriptivo anterior que, si bien no son excesivamente relevantes, sí acaban de redondear el corpus de nuestra explicación.

d) Los grupos $\mathrm{C}$ y $\mathrm{D}$ obtienen medias superiores respecto a los grupos A y B en la prueba de velocidad lectora (VL).

e) El grupo A obtiene medias ligeramente superiores respecto a los grupos $\mathrm{C}$ y $\mathrm{D}$ en las pruebas de lectura silenciosa (LS), comprensión oral $(\mathrm{CD})$ y matemáticas (MAT).

Si observamos detenidamente los resultados obtenidos podemos determinar que éstos son, en gran medida, coherentes con el hecho de que la inmersión linguística es un programa beneficioso para los niños y las niñas de nivel sociocultural bajo a pesar de que no faltan algunos elementos contradictorios. Es decir, los grupos A y B que siguen un programa de inmersión obtienen, en líneas generales, mejo- 
TABLA II

Análisis de varianza para los grupos $A, B, C y D$ respecto la variable $L S$

\begin{tabular}{lrrccc}
\hline Fuente & $\begin{array}{c}\text { Suma de } \\
\text { cuadrados }\end{array}$ & D.F. & $\begin{array}{c}\text { Media } \\
\text { cuadrática }\end{array}$ & F Ratio & Prob. \\
\hline Inter & 113.360 & 3 & 37.787 & 2.810 & .0456 \\
Intra & 954.640 & 71 & 13.446 & & \\
Total & 1.068 .000 & 74 & & & \\
\hline
\end{tabular}

TABLA III

Análisis de varianza para los grupos $A, B, C y D$ respecto a la variable $C O$

\begin{tabular}{lccccc}
\hline Fuente & $\begin{array}{c}\text { Suma de } \\
\text { cuadrados }\end{array}$ & D.F. & $\begin{array}{c}\text { Media } \\
\text { cuadrática }\end{array}$ & F Ratio & Prob. \\
\hline Inter & 71.428 & 3 & 23.809 & 3.678 & .0160 \\
Intra & 459.559 & 71 & 6.473 & & \\
Total & 530.987 & 74 & & & \\
\hline
\end{tabular}

TABLA IV

Análisis de varianza para los grupos $A, B, C y D$ respecto a la variable $M A T$

\begin{tabular}{lccccc}
\hline Fuente & $\begin{array}{c}\text { Suma de } \\
\text { cuadrados }\end{array}$ & D.F. & $\begin{array}{c}\text { Media } \\
\text { cuadrática }\end{array}$ & F Ratio & Prob. \\
\hline Inter & 617.358 & 3 & 205.786 & 3.993 & .0110 \\
Intra & 3658.829 & 71 & 51.533 & & \\
Total & 4276.187 & 74 & & & \\
\hline
\end{tabular}

\section{TABLA V}

Análisis de varianza para los grupos $A, B, C y D$ respecto a la variable $V$

\begin{tabular}{lccccc}
\hline Fuente & $\begin{array}{c}\text { Suma de } \\
\text { cuadrados }\end{array}$ & D.F. & $\begin{array}{c}\text { Media } \\
\text { cuadrática }\end{array}$ & F Ratio & Prob. \\
\hline Inter & 409.238 & 3 & 136.413 & 6.090 & $9.521 \mathrm{E}-04$ \\
Intra & 1.590 .309 & 71 & 22.399 & & \\
Total & 1.999 .547 & 74 & & & \\
\hline
\end{tabular}

TABLA VI

Análisis de varianza para los grupos $A, B, C$ y $D$ respecto a la variable $C I$

\begin{tabular}{lrrccc}
\hline Fuente & $\begin{array}{c}\text { Suma de } \\
\text { cuadrados }\end{array}$ & D.F. & $\begin{array}{c}\text { Media } \\
\text { cuadrática }\end{array}$ & F Ratio & Prob. \\
\hline Inter & $\begin{array}{r}524.126 \\
\text { Intra }\end{array}$ & 3 & 174.709 & 1.088 & .3598 \\
Total & 11.398 .594 & 71 & 160.544 & & \\
\hline
\end{tabular}


res resultados que los grupos $C$ y $D$, que realizan la enseñanza en su lengua materna. Pero los resultados referentes a los dos grupos de inmersión tampoco son parejos. Así, mientras el grupo B se diferencia notablemente de los grupos $\mathrm{A}, \mathrm{C}$ y $\mathrm{D}$ al obtener unas medias significativamente superiores, el grupo A se sitúa sólo un poco por encima de los grupos $C$ y $D$ pudiendo llegar a afirmarse que su rendimiento global es equiparable a éstos.

Nuestra hipótesis, al inicio de este estudio, venía planteada en los siguientes términos: el modelo de inmersión lingüística dirigido a escolares de nivel sociocultural bàjo, si se efectúa adecuadamente, posibilita mejores resultados que si la enseñanza se realiza en la lengua materna. Ya hemos hablado en otro apartado de la enorme importancia que tiene el hecho de que en los programas de inmersión todos los alumnos partan del mismo punto en relación al dominio de la lengua que será vehicular de conocimientos; es decir, su desconocimiento. Ciertamente, garantizada una buena preparación y formación del profesorado, este desconocimiento inicial de la lengua que tienen los niños y las niñas cuando llegan por vez primera a la escuela, se convierte no sólo en una característica más del programa sino en el punto de referencia a partir del cual se va a montar todo el engranaje educativo. De esta forma, un profesorado bilingüe, profundamente consciente de su situación inicial, posibilita algo que de otra forma resulta muy difícil de conseguir: la negociación a partir de cero de todos y cada uno de los significados a compartir en el aula (Cummins, 1981). Esta consideración, aparentemente intrascendente, adquiere una importancia extraordinaria, sobre todo si nos referimos a niños y niñas de nivel sociocultural bajo.

Los trabajos realizados en el campo de la sociología de la educación han mostrado que el lenguaje de estos escolares, desde el punto de vista del significado, difiere del lenguaje que emplean los adultos. Muchos de estos escolares llegan a la escuela con un lenguaje desestructurado y pobre, fruto, en muchas ocasiones, de las escasas oportunidades de mantener interacciones adecuadas cualitativa y cuantitativamente en el ámbito familiar. Es por todo esto por lo que, en estas situaciones, un programa de mantenimiento de la lengua materna puede comportar un alto riesgo de incomprensión entre el lenguaje de la maestra y los alumnos, ya que si bien ambos están utilizando el mismo código, probablemente no pasa lo mismo con los significados implícitos. Por otra parte, como ya hemos dicho, independientemente de cuál sea la lengua materna de los alumnos, el dominio del lenguaje en actividades académicas contextualizadas es justamente lo que posibilita el posterior éxito escolar. Así pues, si la inmersión se realiza adecuadamente tiene que posibilitar el suficiente dominio lingüístico para encarar con éxito las diversas tareas y actividades escolares de los alumnos, ya que la esencia de la inmersión radica precisamente en contextualizar las diferentes actividades que se realizan en el aula así como el lenguaje que las regula.

Además, si tenemos en cuenta las relaciones que se establecen entre la L1 y la L2 tal y como propone la Hipótesis de Interdependencia Lingüística, a las ventajas de la inmersión como el mejor diseño de edu- 
cación bilingüe tenemos que sumar otra: la inmersión dirigida a escolares de nivel sociocultural bajo, por las prestaciones que anteriormente hemos comentado, posibilita mejores resultados que un programa de mantenimiento de la lengua materna si se mantienen en un grado óptimo las condiciones necesarias para que se lleve a cabo. Es decir, la inmersión se revela como un diseño muy potente para promover en estos escolares, a partir de una segunda lengua, el dominio del lenguaje suficiente para afrontar con éxito las tareas académicas propuestas en la escuela. No cabe duda que, con esto, nos estamos refiriendo a las leyes que gobiernan el uso del lenguaje en general, por lo que es de esperar que estos escolares no sólo aprendan una segunda lengua sino que, además, sean capaces a partir de ésta, de mejorar el dominio de su lengua materna.

De esta manera, una vez hechas estas consideraciones, podemos empezar a perfilar de forma más clara la interpretación de nuestros resultados.

En primer lugar tenemos que destacar que no existen diferencias significativas entre los grupos por lo que respecta al cociente intelectual. Esta prueba, basada en rasgos perceptivos, fue escogida con la finalidad de obtener un baremo fiable que nos permitiera conocer de una forma objetiva si los grupos eran comparables entre sí. Hacemos este comentario porque es conveniente destacar aquí que, cuando hablamos en los términos que exponemos en nuestra hipótesis, se podría llegar a interpretar falsamente que en algún momento decimos que la inmersión "vuelve» más inteligentes a los escolares que la siguen. Nada más lejos de esto, lo que posibilita la inmersión lingüística es, por una parte, que desde un principio alumnos/as y maestros/as negocien un código común y compartido en el aula y, por la otra, en tanto que modelo de educación bilingüe, que se favorezca en el escolar la adquisición precoz de determinados aspectos relacionados con habilidades como la conciencia metalingüística, el dominio de la arbitrariedad del signo lingüístico, la «lectura» de imágenes, etcétera.

En segundo lugar tenemos que decir que los resultados obtenidos por el grupo B confirman completamente nuestra hipótesis. Sin embargo, no sucede lo mismo con el grupo A que, recordemos, pertenece a la misma escuela. Este último comentario, aunque quizás parezca poco relevante, sirve para acotar nuestra explicación puesto que, al pertenecer a la misma escuela, quedan descartados todos aquellos factores referentes a diferentes propuestas o proyectos docentes que puedan tener los centros.

La diferencia de resultados entre el grupo A y el grupo B pensamos que puede explicarse a partir de distintos factores: preparación del profesorado, organización de las unidades de enseñanza-aprendizaje, motivación del alumnado hacia el programa, homogeneidad del grupo de alumnos, etc. Sin embargo, no poseemos datos para poder aventurar una explicación ya que se hubiera necesitado un estudio longitudinal en el que, analizándose las unidades didácticas de forma global, se explicitaran las diferencias en cada uno de los grupos. No obstante, si comparamos el rendimiento del grupo A con el de los grupos control podemos observar que, en términos generales, no es diferente. 
Por tanto, sí podemos concluir que para el grupo $A$ el hecho de asistir a un programa de inmersión no se muestra como un factor negativo sino indiferente desde el punto de vista del rendimiento académico global. En tercer lugar, aunque ya hemos dejado claro que no son significativos estadísticamente, cabe resaltar los resultados obtenidos por los grupos experimentales y control en las pruebas de velocidad lectora y de errores en la lectura. Así, podemos observar en términos globales que los grupos A y B tienen medias inferiores en ambas pruebas a las de los grupos $\mathrm{C}$ y D. Sin embargo, tanto el grupo B, significativamente desde el punto de vista estadístico, como el grupo $A$, aunque de forma más ligera, obtienen resultados mejores en las pruebas de comprensión oral, lectura silenciosa y matemáticas. De esta manera, de los resultados se desprende que los grupos A y B que están siguiendo un programa de inmersión, a pesar de que obtienen medias inferiores en las pruebas que miden más directamente los aspectos mecánicos de la lectura y la escritura, por el rendimiento global en las otras pruebas (recordemos que a excepción de la comprensión oral y el test de inteligencia el resto de pruebas necesitan de la lectura para poder ser resueltas exitosamente), podemos concluir que entienden más aquello que leen que los grupos $\mathrm{C}$ y $\mathrm{D}$, que siguen un programa en su propia lengua.

Cabe, finalmente, hacer referencia a la generalización de los resultados. Ya hemos resaltado en diversas ocasiones que debido al tamaño de la muestra, y al carácter exploratorio del estudio, nuestros resultados no tienen la entidad estadística suficiente como para poder derivar afirmaciones concluyentes respecto al resto de la población.

\section{Referencias}

ARNaU, J. (1985). «Educación en la segunda lengua y rendimiento escolar: una revisión de la problemática general». A.M. Siguan (Ed.), Enseñanza en dos lenguas y resultados escolares, Barcelona: Publicacions i Edicions de la Universitat de Barcelona.

Cummins, J. (1979). «Linguistic interdependence and the educational development of bilingual children». Review of Educational Research, 49, 222-251.

Cummins, J. (1981). «The role of primary language development in promoting educational success for language minority students». En Schooling and Langwage Minority Students. $A$ Theoretical Framework, Los Angeles: Evaluation, Dissemination and Assessment Center.

VILA, I. (1983). «Reflexiones en torno al bilingüismo y la enseñanza bilingüe». Infancia y Aprendizaje, 21, 2-23.

VILA, I. (1985). Reflexions sobre l'Educació Bilingüe: llengua de la llar i llengua d'instruccio, Barcelona: Departament d'Ensenyament, Servei d'Ensenyament del Catalá. 


\section{Extended Summary}

The paper briefly reviews the main characteristics which guarantee the success of a home-school language change programme (immersion programme). It also reviews the psychoeducational basis on which they are founded, such as: voluntary participation, bilingual teachers, and students' lack of familiarity with the school language. The author finds that this view supports the application of these type of programmes in schools situated in social and economically deprived areas, the aim being to reduce existing school failure. In this sense the initial hypothesis of this study was put forth in the following terms: If the linguistic immersion model, directed at schoolchildren with low sociocultural status, is carried out correctly, it allows for better results than if instruction is in their mother tongue.

In this study the sample was made up by 2 nd grade students distributed across four groups. Children in two of these groups had been in a Catalan immersion programme from the age of four, and the other two groups had instruction in their mother tongue (Spanish). Both schools were situated in the Carmel district of Barcelona. Students who attend these schools come from families with a low or very low socio-cultural background, their place of birth is Barcelona, and, for the majority, Spanish is their mother tongue. In order to assess the results of this programme, the Department of Experimental Pedagogy of the Faculty of Phylosophy and Educational Sciences, University of Barcelona, prepared a battery of tests. They were complemented with the RAVEN intelligence test and a maths test. The data was analised using the MICROSTA statistical package programme. The number of variables analised were eight: silent reading, oral comprehension, vocabulary, reading speed, errors in reading, written mathematic expression, and IQ.

Even though there are some contradictory elements in the results, they are to a great extent coherent with the fact that language immersion is a programme beneficial to children from low socio-cultural backgrounds. Overall the groups that followed the immersion programme obtained better results than the groups that had instruction in their mother tongue. However, the two immersion programme groups obtained different results. Group B (immersion) showed a marked difference with Groups A (immersion), B and C (mother tongue), as means values were significantly higher, but the results for Group A were only slightly higher than those obtained by Group $C$ and $D$ : The children's general performance here could be said to be comparable. This allows us to be more specific in our initial hypothesis. That is, linguistic immersion is not only not presented as an obstacle for children's linguistic and academic progress, but it may represent a good number of advantages, especially those related with the acquisition of a new language to their linguistic repertoire. But the programme must meet the optimal requirements for putting it into practice. 